\title{
Thrombalexins: Cell-Localized Inhibition of Thrombin and its Effects in a Model of High- Risk Renal Transplantation
}

J. Karegli ${ }^{0,1}$, T.Melchionna ${ }^{0,1}$, C.A.Farrar ${ }^{1}$, R.Greenlaw ${ }^{1}$, D.Smolarek ${ }^{1}$, C.Horsfield ${ }^{1}$, R.Charif ${ }^{2}$, J.H.McVey ${ }^{3}$, A.Dorling ${ }^{1}$, S.H.Sacks ${ }^{1}$ and R.A.G.Smith ${ }^{1 *}$

${ }^{1}$ MRC Centre for Transplantation, King’s College London, Guy’s Hospital, London SE1 9RT, United Kingdom

${ }^{2}$ West London Renal and Transplantation Centre, Imperial College Healthcare NHS Trust, Hammersmith Hospital, London W12 0NN

${ }^{3}$ School of Bioscience \& Medicine, Faculty of Health and Medical Sciences, University of Surrey, Guildford GU2 7XH.

${ }^{0}$ These authors contributed equally to the work

*Towhom correspondence should be addressed: richard.a.smith@kcl.ac.uk, steven.sacks@kcl.ac.uk 


\begin{abstract}
Allograft transplantation into sensitised recipients with anti-donor antibodies results in accelerated antibody-mediated rejection (AMR), complement activation and graft thrombosis. We have developed a membrane-localizing technology of wide applicability that enables therapeutic agents including anticoagulants to bind to cell surfaces and protect the donor endothelium. We describe here how this technology has been applied to thrombin inhibitors to generate a novel class of drugs termed thrombalexins (TLN). Using a rat model of hyperacute rejection we have investigated the potential of one such inhibitor (TLN-1) to prevent acute antibody-mediated thrombosis in the donor organ. TLN-1 alone was able to reduce intragraft thrombosis and significantly delay rejection. The results confirm a pivotal role for thrombin in AMR in vivo. This approach targets donor organs rather than the recipient and is intended to be directly translatable to clinical use.
\end{abstract}

Key Words: accelerated antibody-mediated rejection, coagulation, thrombin, complement, cytotopic, immunosuppression, kidney, membrane-targeting therapy, Thrombalexin, transplant rejection.

Running Title: Thrombalexin in transplantation

Abbreviations: AMR, Antibody-Mediated Rejection; DA, Dark Agouti; GVB: Gelatin-Veronal buffer; MSB, Martius Scarlet Blue; MST; median survival time, PBS, Phosphate Buffer Saline; RBC, Red Blood Cells; TLN, Thrombalexin; TLN-1 (also called PTL004) 


\section{Introduction}

The modification of pharmacologically active agents by specific attachment of generic "cytotopic" reagents based on the myristoyl-electrostatic switch (1) has been described elsewhere (2). The term "cytotopic" is used in this paper to mean a chemically modified agent that acts primarily at the surface (topos) of a cell (cytos). The approach has found particular use with regulators of complement activation, notably fragments of CR1 (CD35) (3), CD55 (4) and CD59 (5). Cytotopic membrane-localising groups are relatively hydrophilic despite containing at least one membrane-insertive alkanoyl group and conjugates of active agents with such groups remain sufficiently water-soluble to be used as (bio)pharmaceuticals (2). Furthermore, such agents can be applied locally to organs or tissues where they are retained and function in a regioselective fashion.

Experimental and clinical experience with the CR1 derivative Mirococept (APT070) has shown that this agent can be delivered to kidneys $(6,7)$ and in one case, human liver (8) by ex-vivo perfusion resulting in uptake by the microvasculature of the organ and a consequent enhanced complement inhibitory activity upon reperfusion of the implanted graft. The process of antibodymediated rejection (AMR) not only involves complement activation, but also the direct effects of high levels of anti-donor MHC Class I and Class II antibodies, which activate donor endothelium promoting intravascular thrombosis; in the florid form seen in hyperacute AMR, this can cause graft necrosis.

Detailed histological evaluation of large animal cardiac allo- and xenografts (and human allografts) undergoing AMR suggests that this thrombotic process, beginning in venules, is a very 
early feature of the rejection process (9). In AMR associated with blood group-incompatible renal transplantation, it was evident that glomerular fibrin deposition was a very early manifestation of humoral rejection, appearing before graft dysfunction in some cases (10). The major benefit of inhibiting thrombin generation after xenotransplantation was shown by our group several years ago using transgenic mouse hearts transplanted into rats, following which the manifestations of AMR were completely abolished, though notably the hearts were still susceptible to cellmediated rejection (11). In xenografts, this phenomenon can arise because xenogeneic endothelial cells are capable of direct conversion of prothrombin to active thrombin $(12,13)$, the central protein in the coagulation cascade triggered via a number of different mechanisms, independently of AMR (14). There are indications that similar complement-independent thrombosis plays a prominent role in rodent AMR models, promoted by molecules such as fgl-2 $(15,16)$ suggesting that targeting coagulation processes directly inside allografts might have similar impact as we have shown in xenografts. We were therefore interested in generating cytotopic anticoagulants, particularly thrombin inhibitors, for use inside organs, specifically to inhibit thrombin generation after transplantation.

In order to target coagulation we constructed a membrane-associated anticoagulant based on a minimized form of hirudin, (a well known thrombin inhibitor, naturally occurring in the salivary glands of leeches). The principle behind the resulting synthetic cell-tethered inhibitor (Thrombalexin, TLN-1) is similar to the complement inhibitor Mirococept (2, 3). TLN-1 binds the phospholipid bilayer using a combination of a bis-myristoyl hydrophobic structure (5) and a lysine-rich sequence, which binds negatively charged phospholipid headgroups. TLN-1, like hirudin and its synthetic derivative Hirulog, interacts with thrombin at the active site and at the fibrinogen-binding exosite I (17). 
In this study, we report the generation and evaluation of TLN-1 in an appropriate rodent model of AMR, testing the hypothesis that treatment of the donor organ with a membrane localizing biological inhibitor of thrombin could prevent the key manifestations of AMR, increasing graft survival, improving renal function and reducing renal tissue injury. 


\section{Materials and Methods}

\section{Animals}

All animals were purchased from Charles River (Kent, UK). Animals used in this project were inbred male DA $\left(\mathrm{RT}^{\mathrm{a}}\right)$ rats, weighing approximately 150g, used as skin and kidney donors. Inbred male Lewis $\left(\mathrm{RT}^{\mathrm{i}}\right)$ rats, weighing approximately $200 \mathrm{~g}$, were used as recipients. Animals had free access to standard rat chow and water. All procedures were carried out in accordance with a UK Home Office license for animal experimentation.

\section{Skin Transplantation}

Based on a method from Guttman (18), Lewis recipients were pre-immunised by sequential grafting of three full thickness tail skin segments $\left(\sim 2 \mathrm{~cm}^{2}\right)$ from DA donors. The skin grafts were held in place by applying a skin adhesive gel (Germolene New Skin, Boots,UK) and bandaged for seven days, after which the bandages were removed. The first two skin transplants were separated by a two-week interval, followed by a four-week period (to allow Ig affinity maturation). 7-10 days after the third skin graft, the Lewis recipients received a DA kidney graft.

\section{Rat Renal Transplantation}

Rat renal transplantation was carried out as described previously (7). The donor and the recipient preparations took place simultaneously thus minimizing the period of donor organ ischemia. Bilateral native nephrectomies were performed, making the survival of the recipient dependent only on the transplanted kidney. Graft rejection was defined either by elevated BUN ( $\geq 40 \mathrm{mM}$ ), or signs of poor health necessitating termination of the experiment, or spontaneous death of an animal, all accompanied by histological evidence of rejection. 


\section{Therapeutic Agents}

TLN-1 (also called PTL004) contains the two thrombin binding sites of Hirulog-1, a structurally minimized form of the leech anticoagulant hirudin (17). This was modified by adding a Cterminal extension to locate a thiol-containing cysteine linker for conjugation at a point distant from either of the two thrombin binding sites to create the peptide we have called 'HLL'. This is used as a non-cytotopic, thrombin-binding agent in some experiments. HLL has the sequence [D-Phe-L-Pro-L-Arg-L-Pro-Gly-Gly-Gly-Gly-L-Asp-Gly-L-Asp-L-Phe-L-Glu-L-Glu-L-Ile-LPro-L-Glu-L-Glu-L-Tyr-L-Leu-Gly-Gly-L-Cys-amide]. HLL was then conjugated with the cytotopic tail APT3146 [(N-alpha, N-epsilon Bis-myristoyl Lys-Ser-Ser-Lys-Ser-Pro-Lys-LysAsp-Asp-Lys-Lys-Pro-Gly-Asp-(S-2-pyridyldithio) Cys], mediated by disulfide interchange (5). The conjugate has a mass of 4649 Daltons by time-of-flight mass spectrometry and was prepared by reaction of the two components in aqueous solution at $\mathrm{pH} \sim 7.4$ followed by further purification (19). The component peptides were prepared using solid phase synthesis by Cambridge Research Biochemicals, Billingham, UK (both components were also synthesized at scale by Almac Sciences, Craigavon, UK). Later variants of TLN have been prepared using different bridging chemistry and will be described elsewhere.

\section{Inhibitory activity of TLN-1 against human thrombin in solution and when tethered to} cells.

Human plasma thrombin (T6884) and N-p-5 Tosyl-Gly-Pro-Arg-pnitroanilide (T1637-substrate) were obtained from Sigma-Aldrich (Gillingham, UK). Stock solutions of 10U/ml and 1mM (respectively) were prepared in $0.05 \mathrm{M}$ Tris- $\mathrm{HCl}, \mathrm{pH} 7.5,0.1 \mathrm{M} \mathrm{NaCl}$ and $0.5 \% \mathrm{BSA}$ (20). Reaction mixtures were prepared with buffer, thrombin (5ul, 0.05U) and substrate (100ul, 
$125 \mathrm{nM}$ ) to a final volume of $800 \mathrm{ul}$ and incubated at $25^{\circ} \mathrm{C}$. When TLN-1 (or control HLL) were included (at various doses), these were premixed with thrombin at $25{ }^{\circ} \mathrm{C}$ for $\sim 7 \mathrm{~min}$ and the reaction was then initiated by addition of substrate. Thrombin-catalyzed hydrolysis of N-p-TosylGly-Pro-Arg-p-nitroanilide was monitored at 405nm on a Spectramax Plus384 (Molecular Devices, Sunnyvale CA,10 USA). The velocity of thrombin-catalyzed reaction was plotted against substrate concentration values using GraphPadPrism5 software and fitting data with nonlinear data matching option. For each substrate concentration assayed, the velocity of the enzyme reaction represented the slope of the linear phase, expressed as amount of product formed per time (A405 vs time). The KM of Thrombin for N-p-Tosyl-Gly-Pro-Arg-p-nitroanilide substrate under these conditions was found to be $10.47+2 \mathrm{nM}$.

Guinea Pig red blood cells (RBC) washed with gelatin-veronal buffer (GVB) were incubated with TLN-1 (or HLL control) for 10min at RT with gentle agitation. After several washes, the supernatant was discarded and the cell pellet resuspended with GVB and thrombin in a final reaction volume of $460 \mathrm{ul}$. Cells were further incubated for $3 \mathrm{~min}$ at $37^{\circ} \mathrm{C}$ in a water bath before being gently pelleted by centrifugation and counted by microscopy. Thrombin activity in the supernatant was assessed as above by addition of T1637-substrate. Values were expressed as percentage of cell inhibition for a certain thrombin amount after subtracting values of HLLtreated cells and normalization for cell number.

\section{Plasma re-calcification assay}

$1.5 \times 10^{5}$ human CD34-derived progenitor cells (21) (kind gift of N Levicar, Hammersmith Hospital) were suspended in $50 \mu$ Tris-buffered saline and mixed with $100 \mu 1$ of normal human plasma (Sigma) in glass tubes (Corning, NY, USA). $100 \mu \mathrm{l}$ of $25 \mathrm{mM} \mathrm{CaCl} 2$ in Tris-buffered saline 
was added and the tube incubated at $37^{\circ} \mathrm{C}$ in a water bath - the time for a fibrin clot to form was determined in triplicate, during which time the tubes were continuously agitated by tilting. In some assays, cells were incubated with $200 \mathrm{uL}$ of HLL or TLN-1 for $2530 \mathrm{~min}$ at $4^{\circ} \mathrm{C}$ before washing twice and inclusion in the clotting assay.

\section{In-Situ Perfusion with TLN-1}

Intra-renal perfusion with TLN-1 $(2 \mu \mathrm{M})$ was based on a previously described protocol (6). TLN1 was dissolved in $5 \mathrm{ml}$ of Soltran perfusion solution (Baxter Healthcare Ltd, UK) perfused into the renal artery (1ml/min over 5 minutes) and left to dwell for ten minutes before flushing with $5 \mathrm{ml}$ of Soltran to wash out unbound TLN-1. The donor kidney was then excised and immediately transplanted into the recipient. Any bleeding from the anastomosis was controlled by Surgicel absorbable haemostat (Ethicon, Somerville, NJ, USA) and gentle pressure.

\section{Collection of Blood and Extraction of Serum}

Blood samples were collected before and after renal transplantation at appropriate time points by tail vein sampling. For serum preparation, whole blood was centrifuged twice for 15 minutes at 14000 rpm. Serum samples taken prior to renal transplantation were used to assess alloantibody titre. Post-transplantation, renal function was assessed by daily estimation of blood urea nitrogen (BUN) using a standard urease kit (BUN Infinity, Fisher Scientific, Loughborough, UK), following the manufacturer's instructions. Terminal blood samples were collected by cardiac puncture. 


\section{Rat splenocyte extraction}

Rat splenocytes were obtained by disruption of fresh spleen through a $70 \mu \mathrm{m}$ sieve into sterile PBS. The cells were centrifuged and incubated with $5 \mathrm{ml}$ of RBC lysis buffer for 5 minutes on ice. Lysis was stopped by adding PBS/ 2\% FCS. The spleen cells were either resuspended in RPMI 1640 (Sigma) or frozen in DMSO (Sigma) in cryogenic vials (Nalgene, UK) and stored in liquid nitrogen.

\section{Detection of Alloreactive IgG}

DA splenocytes were aliquoted (1x10\%/well) into 96 v-well bottom plates, pre-blocked with $50 \mu 1$ of PBS/2\% BSA containing 20\% normal mouse serum and left on ice for 20 minutes. For gating of T-cells for flow cytometric analysis, mouse anti-rat CD3-PE (clone G4.18, eBioscience Ltd) was added to the splenocytes and incubated on for 20 minutes. Serum samples from sensitized recipients were then added at final dilutions of 1:10, 1:50, 1:250, 1:1250 and 1:6250 subsequently incubated as before. Splenocytes were washed twice with PBS/2\% BSA then incubated with mouse anti-rat IgG-FITC (H+L, eBioscience Ltd,) for 20 minutes on ice. Cells were then washed twice and fixed in 2\% paraformaldehyde before being analysed on a FACScan flow cytometer (Becton Dickinson). 


\section{Assessment of Renal Pathology}

Transplanted kidneys removed at appropriate time points were transferred to 4\% paraformaldehyde. Paraffin-embedded tissue was cut into $2 \mu \mathrm{m}$ sections and stained with Martius Scarlet Blue (MSB).

\section{Statistical Analyses}

Values were compared using Student's $t$ test. Survival curves were compared using a log-rank test, and depicted using Kaplan-Meier plots. A difference was considered significant when $\mathrm{p}<0.05$. 


\section{Results}

\section{TLN-1 exhibits membrane-localised inhibition of thrombin and clotting.}

The structure of TLN-1 is shown in Fig. 1A. Guinea Pig RBC were incubated in a solution of TLN-1 $(1 \mu \mathrm{M})$, during which TLN-1 was absorbed onto the RBC surface, so that after washing, the RBC were able to specifically remove $0.05 \mathrm{U}$ of exogenously added thrombin from solution (Fig. 1B). In contrast, RBC incubated with control HLL peptide (see methods) bound no active HLL, such that after washing the RBC were unable to absorb any thrombin. Evidence of nonspecific interaction of thrombin with RBC membrane was not detected in these experiments (data not shown). Dose titration of thrombin revealed that $1 \mu \mathrm{M}$ TLN-1 applied to RBC could remove $80 \%$ of $0.4 \mathrm{U}$ exogenously added thrombin, whereas dose titration of TLN-1 on the RBC revealed that $0.1 \mu \mathrm{M}$ removed $84 \%$ of $0.05 \mathrm{U}$ thrombin (data not shown). These data allowed the calculation of a thrombin inhibitory capacity of about $90 \%$ for $0.1 \mathrm{U} / 10^{4}$ cells exposed to $1 \mu \mathrm{M}$ TLN-1 (typically 1-2 units of thrombin will clot $1 \mathrm{~mL}$ of plasma).

The functional importance of this interaction between TLN-1 and cell membranes is shown in Fig. 1C. Human CD34+ progenitor cells significantly reduced the clotting times of re-calcified human plasma (by virtue of the fact that they expressed human TF (data not shown)), compared to the clotting times observed in glass tubes when no cells were present. Pre-incubation of cells with TLN-1, followed by washing, completely abolished this pro-coagulant activity, but did not inhibit clotting induced by the glass tubes. In contrast, HLL used in equimolar amounts as the TLN-1 and added to the clotting assay, caused significant inhibition of all clotting activity, not just that associated with the presence of the CD34+ cells. 
These data show that TLN-1 binds avidly to cell membranes, binds thrombin and removes it from solution, and exhibits anticoagulant activity selectively at the surface of cells to which it has bound.

\section{Impact of TLN-1 on intragraft thrombosis and graft survival in a sensitised rat renal allograft model of AMR.}

Flow cytometric assessment of DA splenocytes incubated with serum from Lewis rats sensitized by three DA skin grafts revealed high levels of circulating anti-DA IgG (data not shown). These pre-sensitised animals rejected unmodified DA renal allografts with a median survival time (MST) of 1.8 days, compared to control non-sensitised Lewis rats, which rejected with a MST of 5.6 days (Fig. 2A).

Perfusion of DA kidneys pre-transplantation with Soltran alone had no impact on survival time (Fig. 2B) and rat recipients of these kidneys developed a rapid rise in BUN (Fig. 2C\&D). Histology of kidneys harvested at time of rejection revealed florid features of AMR, with 50\% of glomeruli demonstrating thrombosis (Fig. 3 and 4), significant interstitial haemorrhage and infarction of tubules (Figs. 3\&5) and a prominent neutrophil peritubular capillaritis (Fig. 5).

In contrast, perfusion of DA kidneys pre-transplantation with TLN-1 caused a significant prolongation of graft survival, similar to that seen in non-sensitised animals (Fig. 2B), associated with a delayed increase in BUN (Fig. 2C\&D). Histology of rejected kidneys showed a prominent cellular infiltrate, in contrast to the paucity of cells seen in Soltran-perfused kidneys (Figs. 3\&4), and although there were signs of AMR in TLN-1 kidneys, including a marked peritubular capillaritis (Fig. 5), the proportion of glomeruli showing thrombosis was reduced and the 
thrombosis seen tended to be segmental (Fig. 4). With the application of local methods of haemostasis (see methods), there was no evidence of bleeding from the anastomosis in any of the TLN-1-treated grafts, nor of any systemic bleeding tendency in recipients of TLN-1-treated organs.

These data indicate that pre-perfusion of transplanted kidneys with TLN-1 has a significant impact on the mode and timing of rejection in these highly sensitized rats, characterized by inhibition of thrombosis and prevention of infarction, thus allowing time for significant cellular infiltration and the onset of cell-mediated rejection. 


\section{Discussion}

The results presented here demonstrate that a novel compound generated by conjugating a potent direct thrombin inhibitor to a membrane-localizing entity is capable of binding to cell membranes, on which it can selectively capture (and inactivate) thrombin from the milieu and inhibit any procoagulant properties of the cell surface, without apparent impact on clotting induced beyond the antithrombin-treated cell surface. Moreover perfusion of rodent kidney grafts prior to transplantation endowed them with a resistance to the manifestations of AMR, without any overt features associated with systemic anticoagulation. In particular, the impact on intragraft thrombosis was substantial, both in terms of the number of glomeruli that displayed no thrombosis and in the amount of thrombus in that minority of glomeruli with thrombosis. Graft survival was extended to that seen in non-sensitised individuals and was accompanied by a significant cellular infiltrate and extensive tubulitis, consistent with the perception that cellmediated immune mechanisms contributed to rejection in TLN-1 treated organs.

These results are reminiscent of those we presented previously (11), using a non-sensitised xenogeneic model system in which membrane tethered anticoagulants were expressed transgenically on the endothelium of mouse hearts transplanted into rats. Those hearts were rejected by cell-mediated mechanisms despite the presence of high titre anti-mouse antibodies and were completely resistant to intravascular thrombosis, consequent upon sustained and stable expression of a hirudin-based construct by graft endothelial cells. In these new experiments, the effective residence time of TLN-1 on the graft endothelium is probably 24 hours (unpublished data), so it is surprising that the functional impact was so marked, but it illustrates the clinical therapeutic potential of TLN-1 (and later derivatives, unpublished) in situations where a human 
donor organ is at risk of thrombosis, including after desensitisation to remove donor specific HLA antibodies.

Thrombin plays an important role in AMR, not only via generation of fibrin to form occlusive thrombus, but also through other mechanisms, including amplification of the complement cascade via several different routes $(22,23)$ and direct activation of graft endothelial cells via protease-activated receptor signaling (24), which has been shown to be important for local chemokine gradient generation and leukocyte recruitment in the context of AMR (25). Effective control of these non-thrombotic manifestations of thrombin are difficult to achieve using systemic anticoagulants, because of the risk of bleeding associated with the high doses required to maintain inhibition of coagulation proteases at cell membranes (26). Our approach of tethering high concentrations of anticoagulant at the endothelial surface overcomes this potential problem, and it is noteworthy that our results were achieved without significant bleeding from the surgical anastomoses, although local measures including surgical dressings and pressure were necessary. Studies addressing the impact of TLN-1 on complement activation and protease activated receptor signaling will be reported elsewhere.

In summary, the data presented here show that loading of the donor kidney with the novel membrane-adherent anti-thrombin TLN-1 can prevent thrombosis of the graft vasculature without the need for systemic anticoagulation. TLN-1 and its method of use are both translatable to clinical investigation and our current work involves developmental chemistry and toxicology studies to enable subsequent evaluation in phase 1 and 2 studies involving transplant patients at high risk of thrombosis. We envisage that transplant surgery performed using a functionally 
anticoagulated organ without inducing a coagulation defect in the recipient would represent a significant medical advance. In a markedly pro-thrombotic environment, the risk of graft thrombosis will therefore be significantly reduced, at least for the likely duration that the tailed anticoagulant remains on the surface of the endothelial cell.

\section{Acknowledgements.}

We gratefully acknowledge the support of the Medical Research Council of the United Kingdom and of the Wellcome Trust in the further development of this work.

This research was also supported by the National Institute for Health Research (NIHR) Biomedical Research Centre based at Guy's and St Thomas' NHS Foundation Trust and King's College London. The views expressed are those of the authors and are not necessarily those of the NHS, the NIHR or the Department of Health. We also thank the scientific staff of Adprotech Ltd (Cambridge UK, 1997-2004) for their contributions to the development of the cytotopic technology on which the TLN agents are based. 


\section{Figure Legends}

\section{Figure 1: Structure function of TLN-1}

A: Structure of TLN-1 showing the thrombin-binding domain (HLL-upper) and cytotopic (lower) component of the agent.

B: Depletion of thrombin from solution by guinea pig red blood cells incubated with TLN-1 (squares) or HLL (diamonds), before washing, followed by assay of the amount of residual thrombin still present in the supernatant, measured by amidolytic activity using N-p-5 Tosyl-GlyPro-Arg-pnitroanilide.

C: Clotting times of human re-calcified plasma in glass tubes ('No cells'), in the presence of tissue factor-expressing human CD34 cells ('Cells alone'), in the presence of TLN-1-coated human CD34+ cells ('TLN-1 coated') or with human CD34+ cells and HLL in the clotting assay.

\section{Figure 2 Impact of TLN-1 on renal allograft survival.}

A: Actual survival curve following transplantation of DA kidneys into sensitised and nonsensitised Lewis rats. Non-sensitised recipients $(n=6)$ rejected the allogeneic kidneys with a median survival time (MST) of 5.6 days. Recipients pre-sensitised with three DA skin grafts (n=6), rejected kidneys with a MST of 1.8 days.

B: Actual survival curve following transplantation of DA kidneys into sensitized Lewis rats. Control allografts perfused with Soltran pre-transplantation $(n=4)$ rejected kidneys with a MST of 1.5 days. Allografts perfused with Soltran-containing TLN-1 pre-transplantation $(\mathrm{n}=6)$ showed a statistically significant prolongation of survival to 5.1 days.

C: Dynamic changes in renal function post transplantation. Plot of daily BUN estimations from rats in B receiving Soltran-perfused (dotted lines) or Soltran+TLN-1treated grafts (solid line). 
D: Mean ( \pm SEM) day 1 BUN values from Soltran (black bar) vs. Soltran+TLN-1 (white bar) perfused allografts illustrating statistically significant differences in renal function at this time point.

\section{Figure 3: Histology from allografts perfused with Soltran vs. Soltran+TLN-1}

Representative sections from Soltran (A-C) or Soltran containing TLN-1-perfused (D-F) DA kidney allografts harvested on day of rejection. All stained with MSB to highlight fibrin (red) and erythrocytes (yellow) taken at x100 magnification.

A-C: Illustrating thrombosis (black arrows) in 3/5 (A), 4/6 (B) and 4/5 glomeruli (C). Sections also illustrate tubular necrosis, interstitial haemorrhage and polymorphs in peritubular capillaries (area defined by elipse in B).

D-F: Illustrating thrombosis (black arrows) in 0/4 (D), 1/4 (E) and 2/4 glomeruli (F). Sections also illustrate extensive cellular infiltrate (white arrows in E), interstitial haemorrhage (yellow arrow F) and tubular infarction (blue arrow F).

Figure 4 Glomerular thrombosis seen in from allografts perfused with Soltran vs. Soltran+TLN-1

Representative sections from Soltran (A-C) or Soltran-containing TLN-1-perfused (D-F) DA kidney allografts harvested on day of rejection. All stained with MSB to highlight fibrin thrombi (red) and erythrocytes (yellow) and taken at x400 magnification.

A-C: Representative glomeruli from majority (A,B thrombosed) and minority (C) of kidneys perfused with Soltran alone. Examination of 10 low power fields per section revealed average of $53 \%$ of glomeruli contained thrombus. 
D-F: Representative glomeruli from majority (D, E- non-thrombosed) and minority (F) of kidneys perfused with Soltran containing TLN-1. Examination of 10 low-power fields per section revealed average of $24 \%$ of glomeruli contained thrombus.

Figure 5 Inflammatory lesions in from allografts perfused with Soltran vs. Soltran+TLN-1. Representative sections from Soltran (A,B) or Soltran containing TLN-1-perfused (C,D) DA kidney allografts harvested on day of rejection. All stained with MSB and taken at x100 (A, C) or x200 (B,D) magnification.

A\&B: Illustrating the lack of significant interstitial infiltrate but prominent tubular necrosis (A) and extensive polymorphonuclear leukocytic peritubular capillaritis (area defined by elipse in B). C: Illustrating the significant cellular lesions manifesting as tubultis (black arrows), glomerulitis (white arrow) and peritubular capillaritis (red arrows).

D: Higher power image to illustrate the peritubular capillaritis (red arrows). 


\section{References:}

1. Thelen M, Rosen A, Nairn AC, Aderem A. Regulation by phosphorylation of reversible association of a myristoylated protein kinase C substrate with the plasma membrane. Nature 1991;351(6324):320-322.

2. Smith RA. Targeting anticomplement agents. Biochem Soc Trans 2002;30(Pt 6):10371041.

3. Mossakowska D, Dodd I, Pindar W, Smith RA. Structure-activity relationships within the N-terminal short consensus repeats (SCR) of human CR1 (C3b/C4b receptor, CD35): SCR 3 plays a critical role in inhibition of the classical and alternative pathways of complement activation. Eur J Immunol 1999;29(6):1955-1965.

4. White J, Lukacik P, Esser D, Steward M, Giddings N, Bright JR et al. Biological activity, membrane-targeting modification, and crystallization of soluble human decay accelerating factor expressed in E. coli. Protein Sci 2004;13(9):2406-2415.

5. Fraser DA, Harris CL, Williams AS, Mizuno M, Gallagher S, Smith RA et al. Generation of a recombinant, membrane-targeted form of the complement regulator CD59: activity in vitro and in vivo. J Biol Chem 2003;278(49):48921-48927.

6. Patel H, Smith RA, Sacks SH, Zhou W. Therapeutic strategy with a membrane-localizing complement regulator to increase the number of usable donor organs after prolonged cold storage. J Am Soc Nephrol 2006;17(4):1102-1111.

7. Pratt JR, Jones ME, Dong J, Zhou W, Chowdhury P, Smith RA et al. Nontransgenic hyperexpression of a complement regulator in donor kidney modulates transplant 
ischemia/reperfusion damage, acute rejection, and chronic nephropathy. Am J Pathol 2003;163(4):1457-1465.

8. Banz Y, Inderbitzin D, Seiler CA, Schmid SW, Dufour JF, Zimmermann A et al. Bridging hyperacute liver failure by ABO-incompatible auxiliary partial orthotopic liver transplantation. Transpl Int 2007;20(8):722-727.

9. Rose AG, Cooper DK. Venular thrombosis is the key event in the pathogenesis of antibody-mediated cardiac rejection. Xenotransplantation 2000;7(1):31-41.

10. Fidler ME, Gloor JM, Lager DJ, Larson TS, Griffin MD, Textor SC et al. Histologic findings of antibody-mediated rejection in ABO blood-group-incompatible living-donor kidney transplantation. Am J Transplant 2004;4(1):101-107.

11. Chen D, Weber M, McVey JH, Kemball-Cook G, Tuddenham EG, Lechler RI et al. Complete inhibition of acute humoral rejection using regulated expression of membrane-tethered anticoagulants on xenograft endothelium. Am J Transplant 2004;4(12):1958-1963.

12. Jurd KM, Gibbs RV, Hunt BJ. Activation of human prothrombin by porcine aortic endothelial cells-a potential barrier to pig to human xenotransplantation. Blood Coagul Fibrinolysis 1996;7(3):336-343.

13. Siegel JB, Grey ST, Lesnikoski BA, Kopp CW, Soares M, Schulte am Esch J, 2nd et al. Xenogeneic endothelial cells activate human prothrombin. Transplantation 1997;64(6):888-896.

14. Fenton JW, 2nd, Ofosu FA, Moon DG, Maraganore JM. Thrombin structure and function: why thrombin is the primary target for antithrombotics. Blood Coagul Fibrinolysis 1991;2(1):6975.

15. Ning Q, Sun Y, Han M, Zhang L, Zhu C, Zhang W et al. Role of fibrinogen-like protein 2 prothrombinase/fibroleukin in experimental and human allograft rejection. J Immunol 2005;174(11):7403-7411. 
16. Mendicino M, Liu M, Ghanekar A, He W, Koscik C, Shalev I et al. Targeted deletion of Fgl-2/fibroleukin in the donor modulates immunologic response and acute vascular rejection in cardiac xenografts. Circulation 2005;112(2):248-256.

17. Maraganore JM, Bourdon P, Jablonski J, Ramachandran KL, Fenton JW, 2nd. Design and characterization of hirulogs: a novel class of bivalent peptide inhibitors of thrombin.

Biochemistry 1990;29(30):7095-7101.

18. Guttmann RD. Genetics of acute rejection of rat cardiac allografts and a model of hyperacute rejection. Transplantation 1974;17(4):383-386.

19. Smith RAG, Sacks SH, Dorling A, Inventors; Antithrombotic compounds patent US 2012232014 A1 2011.

20. Lottenberg R, Christensen U, Jackson CM, Coleman PL. Assay of coagulation proteases using peptide chromogenic and fluorogenic substrates. Methods Enzymol 1981;80 Pt C:341-361. 21. Gordon MY, Levicar N, Pai M, Bachellier P, Dimarakis I, Al-Allaf F et al. Characterization and clinical application of human CD34+ stem/progenitor cell populations mobilized into the blood by granulocyte colony-stimulating factor. Stem Cells 2006;24(7):18221830.

22. Huber-Lang M, Sarma JV, Zetoune FS, Rittirsch D, Neff TA, McGuire SR et al. Generation of C5a in the absence of C3: a new complement activation pathway. Nat Med 2006;12(6):682-687.

23. Krisinger MJ, Goebeler V, Lu Z, Meixner SC, Myles T, Pryzdial EL et al. Thrombin generates previously unidentified C5 products that support the terminal complement activation pathway. Blood 2012;120(8):1717-1725.

24. Shrivastava S, McVey JH, Dorling A. The interface between coagulation and immunity. Am J Transplant 2007;7(3):499-506. 
25. Chen D, Carpenter A, Abrahams J, Chambers RC, Lechler RI, McVey JH et al. Proteaseactivated receptor 1 activation is necessary for monocyte chemoattractant protein 1-dependent leukocyte recruitment in vivo. J Exp Med 2008;205(8):1739-1746.

26. Ahamed J, Belting M, Ruf W. Regulation of tissue factor-induced signaling by endogenous and recombinant tissue factor pathway inhibitor 1. Blood 2005;105(6):2384-2391. 
Figure 1
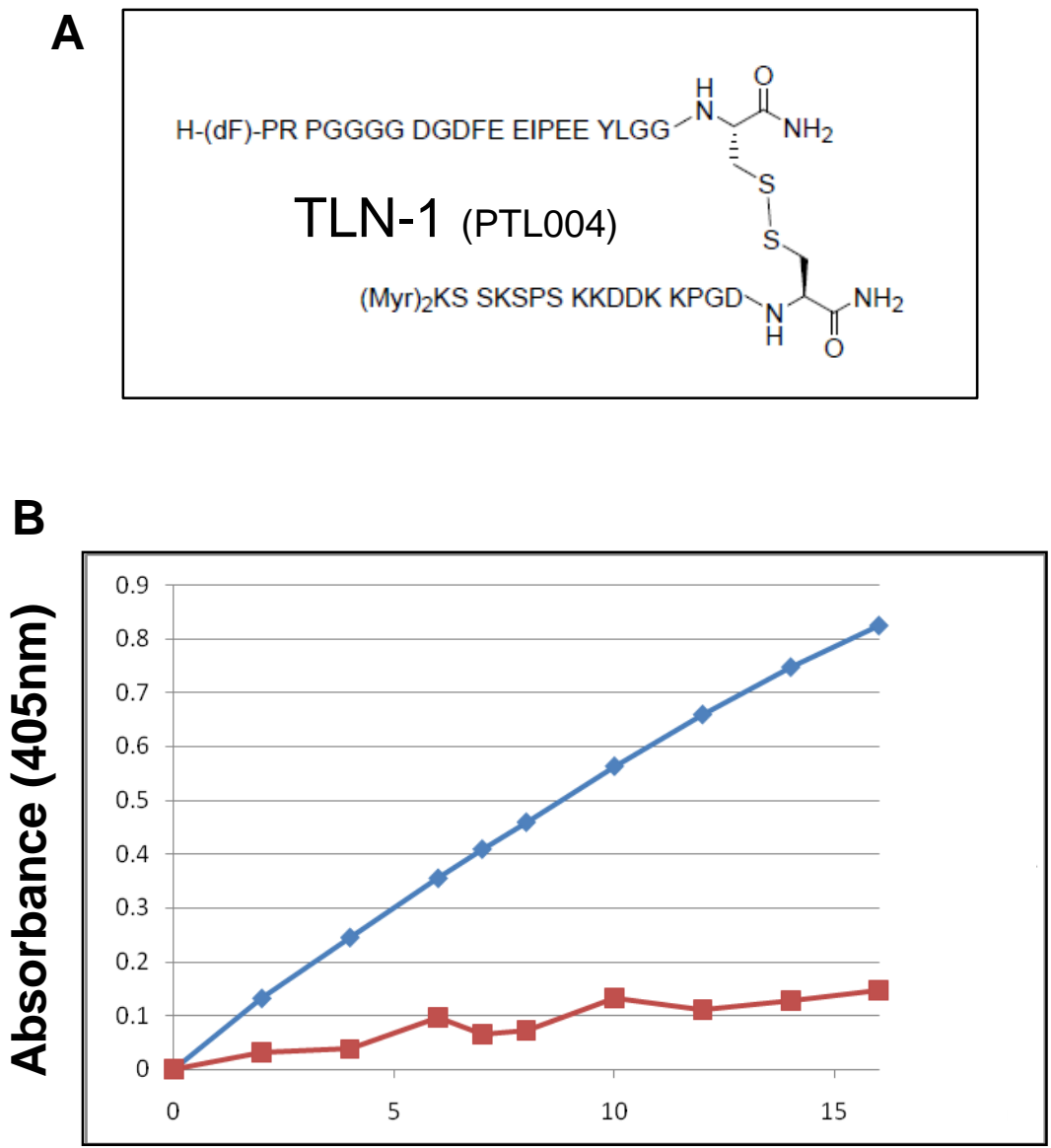

Time (mins)

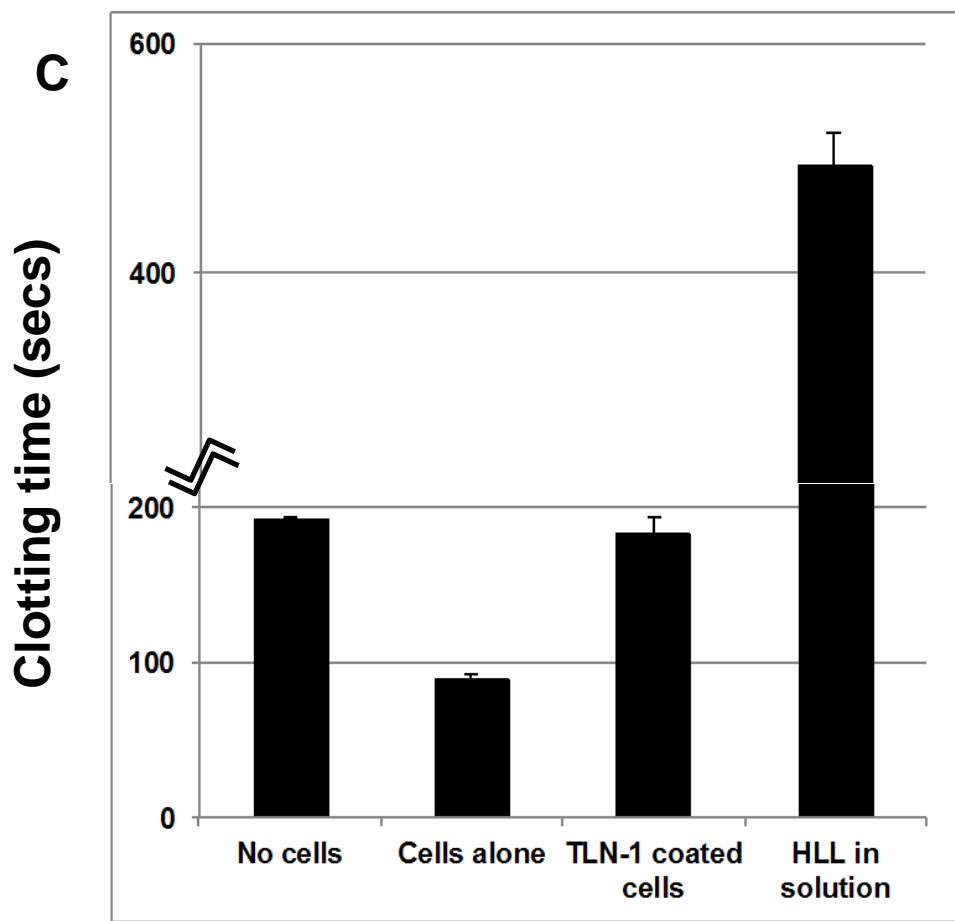


Figure 2

A

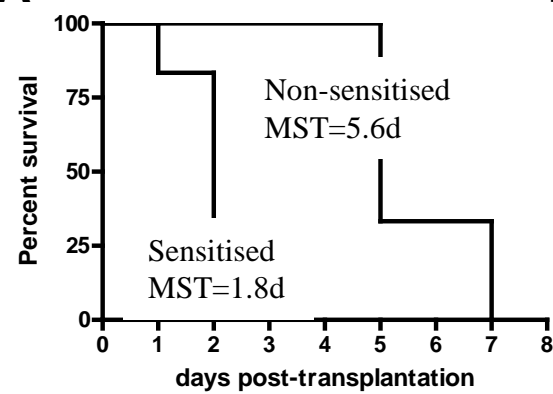

C

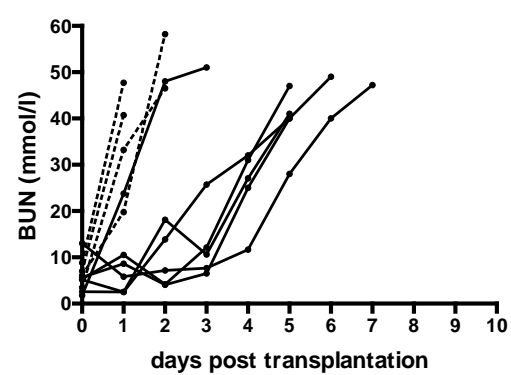

B

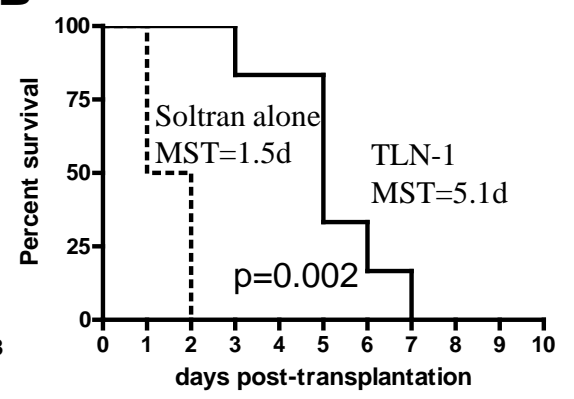

D

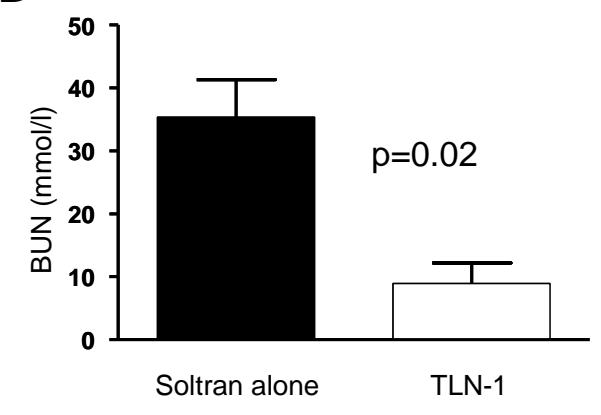


Figure 3
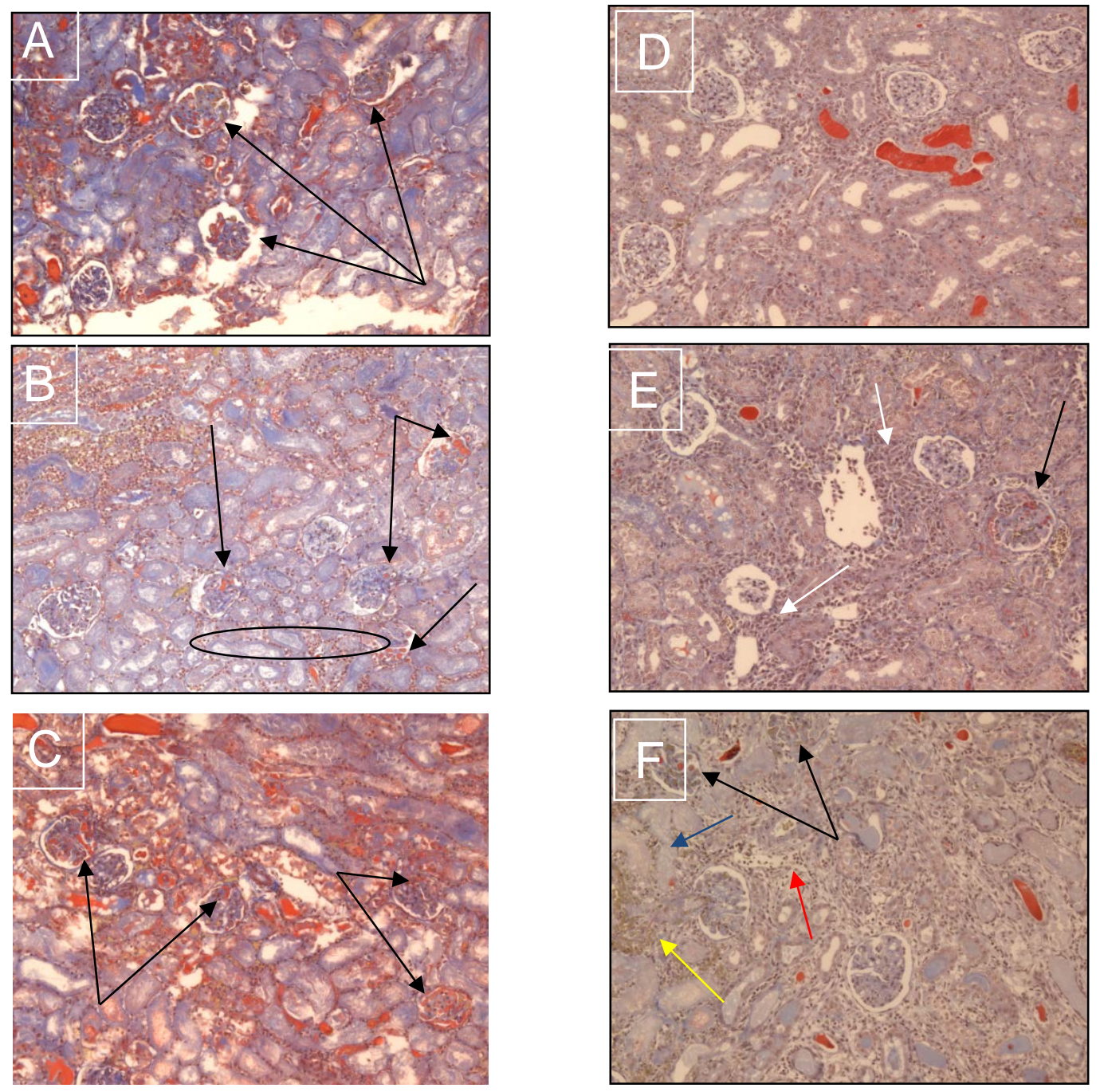
Figure 4
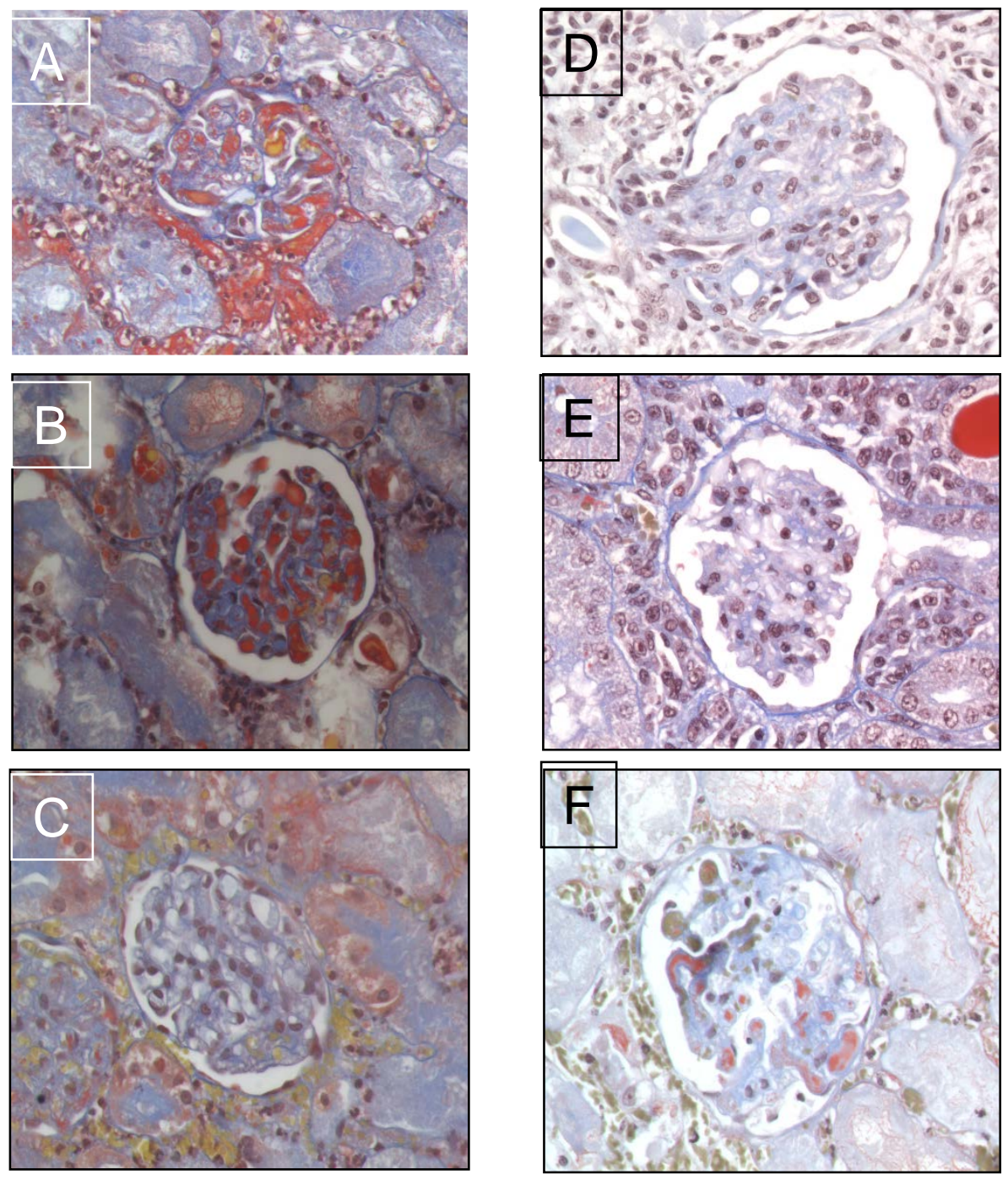
Figure 5
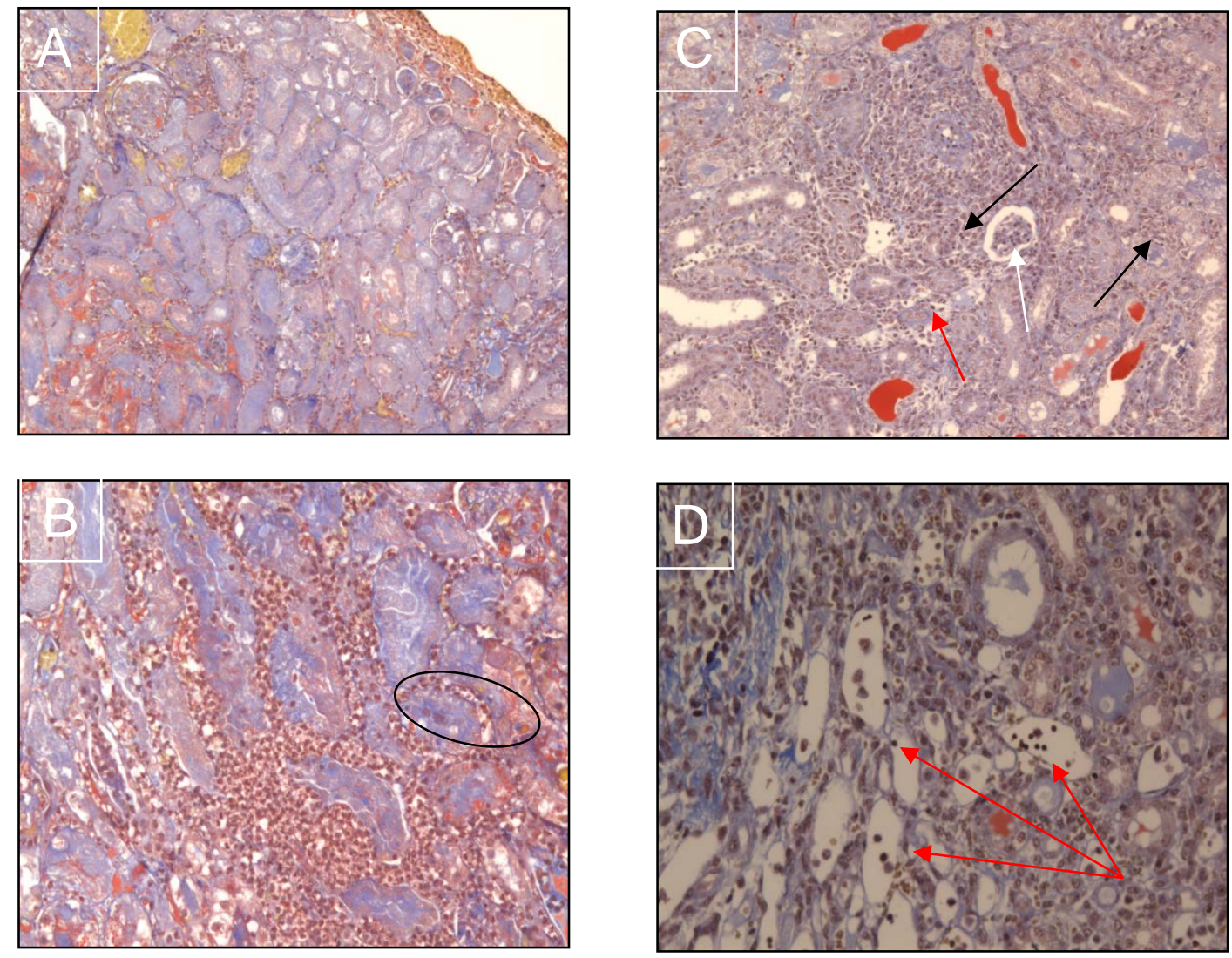